\title{
EBP: An Efficient Broadcast Protocol for Warning Message Dissemination in VANETs
}

\author{
Ouafa Mahma ${ }^{1}$, Ahmed Korichi ${ }^{1}$ and Abdelhabib Bourouis ${ }^{2}$ \\ ${ }^{1}$ Kasdi Merbah University, Ouargla, Algeria \\ ${ }^{2} \operatorname{ReLa}(\mathrm{CS})^{2}$ Lab, University of Oum El Bouaghi, Algeria
}

\begin{abstract}
Vehicular Ad-hoc Networks (VANETs) enable vehicle-to-vehicle communications to share relevant road information with various applications. Road safety is one of the main objectives of VANETs that has attracted great interest from researchers. Other so-called comfort applications have also been studied which can improve driving experience and passenger safety. In all these applications, sharing of warning messages can help drivers minimize accidents and congestions, and plan better itinerary during the congestion situations or the anticipation of potential and highly hazardous events. In this paper, we present an efficient broadcast protocol (EBP) for broadcasting warning messages in VANETs. As an improved strategy for alert data dissemination, EBP can be applied in whatever the nature of the risk, with a particular focus on mobile dangers. We first conduct an in-depth analysis and evaluation, under different conditions, of the existing approaches and mechanisms used for information dissemination in VANETs. Then, we point out their drawbacks and design the EBP to avoid these drawbacks. As a result, the EBP is an improved, well-justified and more effective protocol. We validate it by simulation experiments under various scenarios.
\end{abstract}

ACM CCS (2012) Classification: Networks $\rightarrow$ Network type $\rightarrow$ Adhoc networks

Networks $\rightarrow$ Network performance evaluation $\rightarrow$ Network simulations

Keywords: VANET, mobile risk, data dissemination, simulation, performance measuring

\section{Introduction}

To address the dilemma between high dependence of people's lives on means of transportation and the growth of traffic problems, thanks to technological advances, it is possible nowadays to provide vehicles with various sophisticated road navigation equipment, based on which VANETs can be formed. These dynamic networks allow sharing data in a collaborative way among intelligent vehicles that can communicate in two forms, vehicle to vehicle (to each other) and vehicle to infrastructure (roadside units).

Due to the significant increase in the number of vehicles in circulation and the rate of road accidents, it is necessary to control road traffic in order to ensure safe road navigation. VANETs can play a critical role for this purpose, where vehicles broadcast important messages to each other so as to improve road safety.

Many broadcasting protocols have been developed for VANETs. They can be classified into two basic categories [1], [2]:

- Single-hop broadcast protocols: Instead of flooding the network while traveling, each vehicle shares periodically with its onehop neighbors the packets to broadcast [3].

- Multi-hop broadcast protocols: Information is spreaded in the network by means of flooding. When the source vehicle broadcasts an information packet, a number of vehicles within the proximity become the relay vehicles which rebroadcast the packet further in the network. Similarly, after these relay vehicles (nodes) rebroadcast the packet, the vehicles in their vicinities will become the next tier relay nodes and 
perform the task of forwarding the packet further. As a result, the information packet is able to propagate from the sender to almost all other distant vehicles. Dangerous accidents require immediate notification dissemination to ensure safety on the road. Existing solutions proposed for VANETs make use of various concepts to design broadcasting protocols and to get the best results for a specific need. However, they are mainly focused on immobile risks occurring in a given region with fixed coordinates. There is less study of their effectiveness in the case of dynamic/mobile risk. Although this is not a new problem, it is somehow missed or neglected in existing works. In contrast, in this paper, we are interested in this kind of real situations for better consideration and characterization of dangerous events. The following two examples can illustrate our idea in this paper:

- A traffic accident resulted from the loss of control of a truck carrying flammable liquid. The loss of control caused slippage of the truck on a highway where there were dozens of vehicles traveling at very high speeds (can exceed $100 \mathrm{~km} / \mathrm{h}$ ). (Algeria, 2013), [2].

- An accident caused by the dysfunction of a device known as "speed regulator" which blocks the speed of a vehicle at 200 $\mathrm{km} / \mathrm{h}$ (on the road from France to Belgium, 12/02/2013), [2].

The lack of real-time information dissemination in these accidents led to catastrophic damages.

Based on these examples, we will first study the effectiveness of the existing data dissemination mechanisms. Then, we will propose a protocol with the focus on achieving a timely diffusion of the safety information in mobile danger situations. The optimality of our proposed protocol will be studied in terms of the delay of safety data dissemination and the efficiency of the dissemination strategy in both stationary and mobile risks.

The rest of this paper is organized as follows. Literature review on safety data dissemination protocols in VANETs with a discussion of their performance is presented in Section 2. Description of the proposed protocol is given in Sec- tion 3. Operational mode of the protocol and the corresponding algorithm are discussed in sections 4 and 5. In Section 6, a performance evaluation by means of simulation is presented. Finally, the conclusion with perspectives and future enhancements is given in Section 7.

\section{Related Work}

Research and development efforts devoted to VANETs in recent years are very impressive. This has been motivated by the growing demand in road safety as well as the very tight competition on the market for this type of applications in automotive industry.

In our previous work [2], [4], [5], we focused on multi-hop broadcasting protocols and particularly on the approaches to improve temporal or spatial delay measures. In the meantime, we have developed broadcast protocols based on position, probability, location or distance. In this paper, we will emphasize the computation of the optimal probabilities in relay selection. Before discussing our approach, we provide a review of the advantages and weaknesses of some recent and pertinent related works.

\subsection{Position-Based Protocols}

The Urban Multi-hop Broadcast (UMB) protocol [6] tried to solve the broadcast storm and the hidden node problems that happen due to the high density of vehicles in urban environments. However, the UMB protocol does not guarantee the absence of packet collision as this protocol allows more than one vehicle in the same segment and these vehicles can send the control message named CTB (clear to broadcast packet) at the same time. In addition, this protocol requires some time for the selection of the next transmitter, which makes the delay unpredictable and could be very long. Therefore, it is inappropriate for real time applications.

There are other similar protocols based on the same principle, with some differences in underlying techniques such as AMB (Adhoc MultiHop Broadcast) [7] [6] and multi-hop broadcast protocol for emergency message disseminations in urban vehicular networks (UMBP) [7] [6]. AMB is an extension of UMB for broad- 
casting at intersections. UMBP is intended to reduce the amount of redundant data and the end-to-end delay [8].

The Geographic Random Forwarding protocol (GeRaF) [9] uses the geographical position of the recipient instead of its network address. The operating principle of this protocol, and other protocols of this category, is based primarily on the geographic destination sector specified by the source. The data is forwarded to the destination by intermediate relay nodes. Election of a relay is conducted according to its position compared to the destination. This raises the problem of collision, which increases proportionally with the number of vehicles, since the intermediate node receiving the message in a broadcast manner is not obliged to act as a relay. A node may decide to become a relay according to its relative position to the destination.

In order to improve the proposed technique, the Position Based Adaptive Broadcast protocol (PAB) [10] adopts a certain waiting time before making the decision to be a relay vehicle. Upon the timeout of the specific backoff and if no one has already forwarded the message, the vehicle becomes a relay and retransmits the message to neighbors. Contrary to the SB (Smart Broadcast) protocol [11], which is based only on the distance between the transmitter and the receiver in the next relay selection, PAB proposes a formula combining vehicle position and speed.

\subsection{Distance-Based Protocols}

The Smart Broadcast (SB) protocol [11] is another improvement of UMB in terms of latency, waiting time (to assign the next relay with a delay function (WR)) and overhead. The message propagation speed is improved compared to UMB and GeRaF even in the case of high network density. The Efficient Directional Broadcast (EDB) protocol [12] works on the same principle as UMB to resolve the problem of high number of redundant retransmissions. It designs a delay for each node in the transmission range before the rebroadcast. The duration of the delay, computed according to the distance between the vehicle and the transmitter, is specific for each vehicle.

In the Reliable Method for Disseminating Safety Information (RMDSI) protocol [12], [13], the authors use the delay to determine the retransmission priority for each vehicle. Following the same principle as EDB, after receiving a packet, the vehicle waits for some time before rebroadcasting it. Upon expiration of the waiting time, the vehicle retransmits the packet. If vehicles receive duplicated messages before their waiting time expiration, the broadcast phase is cancelled. In simulations when the network is highly fragmented, RMDSI is more efficient than UMB, since the latter does not consider the fragmentation problem. In the Reliable Broadcasting of Life Safety Messages (RBLSM) protocol [14], the priority in selecting a relay is given to the vehicle nearest to the transmitter. It is assumed that the less the distance between the vehicle and the transmitter, the higher the received signal intensity. This protocol also uses control packets RTB and CTB.

The Multi-hop Vehicular Broadcast protocol (MHVB) [15] also exploits the waiting time before rebroadcasting, where the vehicle farthest from the transmitter always has the shortest waiting time. This time is calculated by an allocation function, which is not explicitly stated. However, this protocol detects traffic congestion based on two indicators: the number of neighbors of each vehicle and their speed. The Opportunistic broadcast of warning messages in VANETs with unreliable links (OppCast) protocol [16] aims to reduce the number of transmissions, while offering high network packet reception ratio (PRR) and improving the propagation speed simultaneously. A double-phase broadcast strategy is proposed to obtain fast message propagation and to ensure high PRR. The idea of opportunistic forwarding is operated at each hop to reduce the propagation latency. This protocol is designed as a MAC-layer broadcast coordination function, which allows multiple nodes to agree on the actual relay nodes in a distributed way. It can mitigate the hidden terminal problem. Simulation results show that, under various vehicle densities, OppCast achieves almost 100\% PRR and fast dissemination rate, while using significantly fewer number of transmissions compared with the existing competing protocols [14] [16].

The Abiding Geocast protocol (AG) [17] is a geocast inter-vehicle communication system for warning massage dissemination in VANETs. It is proposed to increase the probability of access 
to all relevant vehicles and to reduce the overhead. To inform a set of vehicles in a specific geographical area about a nearby risk, the first vehicle that detects the risk starts broadcasting the warning message to other vehicles to inform them of this dangerous situation. The authors used various dissemination strategies, including (1) use the vehicle as a reverse relay, and (2) update the waiting time dynamically for the next release.

The Optimal Multi-hop Broadcast protocol for vehicular safety (OCAST) [18] is an optimization protocol for warning message dissemination in VANETs to secure roads. It optimizes time and number of transmissions, which is conducted using a dissemination strategy that exploits the opposed vehicles as relays. It uses waiting times to complete the coordination of reliable and efficient distributions. It elects the farthest vehicles as relays to reduce data redundancy in the network [16].

\subsection{Probability-Based Protocols}

The Optimized AdaPtive Broadcast scheme for Inter-vehicle communication (OAPB) [19] is based on beacon messages. It assigns a shorter delay time for vehicles having larger probabilities that are calculated based on the number of neighbors within two hops. The Reception Estimation Alarm Routing protocol (REAR) [20] uses the reception probability to select the next relay. Only the vehicles in the message propagation direction calculate this probability. The vehicle with the least contention delay is selected as the relay vehicle [21]. The Irresponsible Forwarding protocol (IF) [22] is based on the distance of vehicles from the source and the neighbors density. Each node implicitly evaluates the probability of another existing node, which will make the rebroadcast more efficient. The node does not rebroadcast when this probability is sufficiently high.

Generally, all these protocols are developed by improving the existing protocols, either in time or in performance. They can be organized into: single-hop or multi-hop categories, depending on the methodology used in safety data dissemination and the scheme used in solving the broadcast storm problem.

\section{System Description and Assumptions}

In this paper, we present a multi-hop broadcast protocol, which is an optimal and reliable scheme for data dissemination based on probability and distance. The main objective is to inform, within a fairly short period of time, a group of vehicles in a specific area about a mobile danger in the vicinity. This new protocol is developed based on the observation that the existing related works did not address sufficiently the case of mobile risk, which may have a more significant impact, especially in the cases where the risk speed is variable or unstable. This protocol can work in the most dangerous scenarios, where vehicles have very high and increasing speed. In our previous work, we used the distance from the dangerous vehicle as a parameter in the development of the corresponding protocol [5]. In this paper, other parameters, such as risk mobility, are also considered to improve effectiveness and performance.

The protocol is called Efficient Broadcast Protocol for Warning Messages in VANETs (EBP). It focuses on improving the transmission time and the number of transmissions so as to reduce communication overhead and to achieve better result for safety data dissemination in VANETs.

This protocol is developed under the following set of assumptions.

- Assumption 1: in the system, there is only one source "initiator". The initiator is the hazardous vehicle, which is the first node that detects the risk and the first node that informs the others by warning message dissemination. The others could act only as relays, silent or inactive.

- Assumption 2: the road is bidirectional (two-way movement), with the possibility of overtaking a single vehicle on the road.

- Assumption 3: vehicles move at allowed and constant speeds that are selected randomly.

- Assumption 4: vehicles are equipped with positioning devices.

- Assumption 5: only one active warning message for a given danger is broadcasted at any point in any time. The message is related to a unique source vehicle. 
- Assumption 6: vehicles are scattered randomly with certain spatial density and the minimal distance between two vehicles is $5 \mathrm{~m}$.

- Assumption 7: the transmission range is fixed at $250 \mathrm{~m}$ for each vehicle.

In most existing protocols, the farthest neighbor node will be responsible for rebroadcasting the alert message [22]. In the probabilistic approaches discussed in Section 2, the rebroadcasting probability is related to the distance between the receiver vehicle and the transmitter vehicle. The farthest vehicle from the source has the highest probability. Our proposed protocol also applies such a probabilistic approach based on the distance between vehicles, especially the distance from the mobile risk.

Specifically, our protocol uses the simple function used in [22] to assign the rebroadcast probability $P$ with a small adjustment, i.e.,

$$
P=\frac{d}{R}
$$

where, as illustrated in Figure $1, P$ is the rebroadcast probability of the neighbor $(b)$ of the vehicle $(a), R$ is the transmission range of the source vehicle $(a), d$ is the mean distance between the source vehicle $(a)$ and the receiver vehicle $(b)$, from time $t_{1}$ to $t_{2}$.

The relay node is selected according to the variation of the distances in two different times $t_{1}$ and $t_{2}$ under the form of two messages (Query/ Response). The probability of rebroadcasting increases proportionally with the mean distance $d$.

In EBP, the selection of the next rebroadcast vehicle is based on the following conditions:

Condition 1:

$$
\operatorname{direction}(a) \neq \operatorname{direction}(b)
$$

Condition 2:

$$
P_{b}=\max \left\{P_{1}, P_{2}, P_{3}, \ldots, P_{n}\right\}
$$

where $n$ is the number of (neighbors of $(a) \cap$ neighbors of $(b))$.
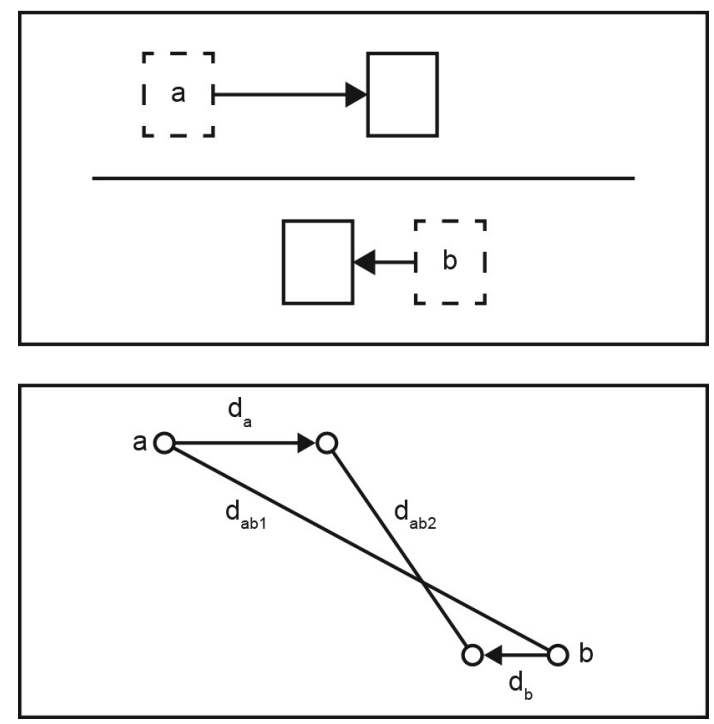

Figure 1. Overview of the dynamic changes in distance of the moving vehicles.

\section{Dissemination Process}

For each new dissemination of the safety message $(x)$, just one vehicle is responsible for triggering this operation. The form of the safety message $(x)$ is:

Message :< warning information,

Sender-information $>$

Warning information : <Initiator-ID, topic, content, speed, location, direction, danger Life Time, Relay-ID >

Sender-information: <ID, direction, speed, location, send Time $>$

1. The source $(a)$ must inspect its direct vicinity with a Beaconless Scheme to detect its neighbors in two consecutive times $t_{1}$ and $t_{2}$. In the Beaconless scheme, the source (a) first broadcasts a message "Query" to its neighbors. Each neighbor, which receives this query, responds by sending the "Response" message informing the source about its position, speed, and direction.

2. All neighbors detect the relative position of the source $(a)$ by verifying the conditions (1) and (2).

3 . If the Condition (1) is met, $P$ (the rebroadcast probability of the selection algorithm) is calculated for all the $n$ neighbors. If $(b)$ has the highest value calculated among all $n$ neighbors, then $(b)$ is the new relay. 
4. The safety message is then sent from $(a)$ to all neighbors. In addition to the information about the danger, this message $(x)$ contains the new sender (Relay-ID). The vehicle $(b)$ - as well as all neighbors - receives the message from the source $(a)$, so it is active and becomes the new relay node.

5. Vehicle $(a)$ receives a "Stop" message from vehicle $(b)$ to cease broadcasting the message $(x)$. Vehicle $(b)$ uses the same protocol to rebroadcast the security message again until the risk stops.

6. When the danger disappears before the expiration of the warning message lifetime, the source vehicle $(a)$ receives a "Stop" message from the corresponding vehicle (initiator) to stop the broadcasting operation.

In [22], it is suggested that, when used in safety applications, beacons should have lower priority than alert messages. Additionally, neighbors do not forward the beacons. However, the information contained in these messages is useful for vehicles to improve their knowledge about their broadcast area and to take decisions appropriately. Especially, the conditions (1) and (2) can be verified by broadcasting a query message. All direct neighbors will reply to this message.

We propose discovering neighbors twice for two reasons. The first is to make sure that the vehicle is not out of the transmission range of the source and the neighboring group (avoid fragmentation). The second is to:

- Verify that there is no error in the received information;

- Ensure the integrity of the information contained in the message to avoid unknown recipients;

- Ensure that the warning message is not lost, which may happen when the newly selected relay leaves a circle without reporting the danger.

The motivation behind using the distance $d_{a b}$ (between $a$ and $b$ ) is that, for the sake of ensuring that all concerned vehicles are informed and the message is not lost, the computation of $d_{a b}$ as a parameter is the solution to keep the message in the effect area.
As we have noted, the source must inspect its direct vicinity with the Beaconless Scheme. This scheme exploits Query/Response technique, which can reduce message collision and network overhead by avoiding redundancy of the broadcast process. According to the parameter $d_{a b}$, the decision to rebroadcast a message is related to the distance between the risky and the recipient vehicle. In particular, the relay vehicle rebroadcasts the message $(x)$ only when the distance $d_{a b}$ is large enough. This guarantees continuity of the process, as mentioned in steps (2) and (3).

\section{State Change Algorithm}

The main objective of our proposed protocol is to guarantee the timely dissemination and the accessibility of warning information for all vehicles in the network. Figure 2 shows an automaton with different states that reflect the behavior of each vehicle in the network.

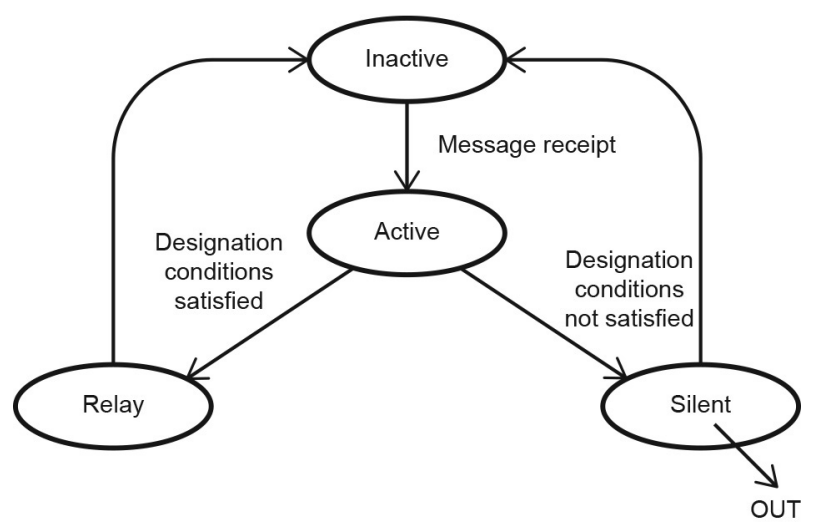

Figure 2. Vehicle behavior according to EBP.

The definitions of the states are listed below:

- Inactive: an oblivious vehicle, which is not yet informed.

- Active: an informed vehicle, which receives a warning message.

- Relay: a vehicle highlighted by the source to rebroadcast the warning message.

- Silent: a vehicle that receives the warning message and has not been designated to be the next relay. 
Generally, a vehicle returns to the "inactive" state from the "silent" state, just after the designation of another relay. It is always possible in this case to participate in the next process of broadcasting (possibility of being chosen later as relay). In the same way, the current relay becomes "inactive" after the designation of the next relay and its confirmation. This process will continue, but may also go "OUT" after a period of time.

\section{Performance Evaluation}

\subsection{Simulation Environment and Parameters}

To study and analyze EBP performance by simulations, we used:

- OMNET ++ (Version: 4.3.1)

- SUMO (Version: 0.17.0)

- Veins (Version: 2.2)

Simulation experiments were conducted using the following parameters: The transmission range was fixed at $250 \mathrm{~m}$. The safety distance was $250 \mathrm{~m}$. Traffic volume was set between 200 and 700 (vehicle/hour). The simulation experiment duration was fixed to $6000 \mathrm{~s}$. The danger occurred at 400 seconds, which triggered the dissemination of the warning message. In simulations, the measured parameters are the percentage of informed vehicles, the number of delivered messages and the probability of informing all vehicles in the network in different cases.

\subsection{Results and Analysis}

The following tables and figures show information about the nodes that are extracted from the trace file generated in several simulation scenarios:

Figure 3 is a snapshot of the graphical animation under OMNET ++ . Vehicles are represented as colored balls. The light gray ones refer to the informed (about the risk) vehicles (in "silent" or "active" states) and the dark gray ball on the left to the dangerous vehicle. In the middle it represents the actual source, and rightmost dark gray ball is the next relay.

Tables 1 and 2 show some statistics obtained from simulation trace files of these scenarios.

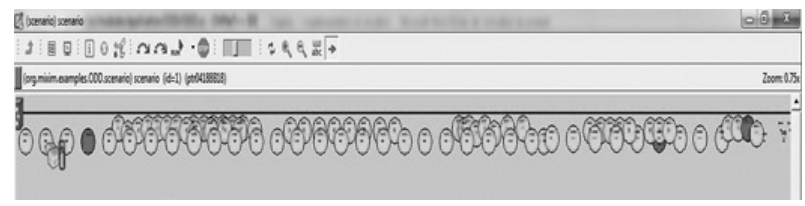

Figure 3. Selection of the best next relay and rebroadcast of the warning message

(OMNET++ scenario).

Table 1. Risk changes vs. informed vehicles numbers.

\begin{tabular}{|c||c|c|c|c|c|c|}
\hline Case & $\begin{array}{c}\text { Number of } \\
\text { relays }\end{array}$ & $\begin{array}{c}\text { Total number of } \\
\text { vehicles }\end{array}$ & Risk speed & $\begin{array}{c}\text { Number of } \\
\text { informed vehicles }\end{array}$ & Risk life-time & $\begin{array}{c}\text { Simulation } \\
\text { duration }\end{array}$ \\
\hline \hline Case 1 & 07 & 1000 & $20 \mathrm{~m} / \mathrm{s}$ & 115 & $420 \mathrm{~s}$ & $6000 \mathrm{~s}$ \\
\hline Case 2 & 09 & 1200 & $20 \mathrm{~m} / \mathrm{s}$ & 120 & $600 \mathrm{~s}$ & $6000 \mathrm{~s}$ \\
\hline Case 3 & 11 & 1200 & $25 \mathrm{~m} / \mathrm{s}$ & 125 & $900 \mathrm{~s}$ & $6000 \mathrm{~s}$ \\
\hline
\end{tabular}

Table 2. Informed vehicles rate vs. number of relays.

\begin{tabular}{|c||c|c|c|c|}
\hline Case & $\begin{array}{c}\text { Number of relays } \\
\text { (selected vehicles) }\end{array}$ & $\begin{array}{c}\text { Rates of neighboring vehicles } \\
\text { informed before reaching the } \\
\text { risk zone }\end{array}$ & $\begin{array}{c}\text { Density of traffic } \\
\text { (vehicle/km/lane) }\end{array}$ & $\begin{array}{c}\text { Total number of } \\
\text { informed vehicles }\end{array}$ \\
\hline \hline Case 1 & 07 & $100 \%$ & $1-15$ & 115 \\
\hline Case 2 & 09 & $100 \%$ & $1-15$ & 120 \\
\hline Case 3 & 11 & $100 \%$ & $1-15$ & 125 \\
\hline
\end{tabular}


Table 1 shows various parameters: network vehicles number, risk lifetime and risk speed, the total number of informed vehicles and the number of engaged relays. Table 2 shows the optimal delivery rate of $100 \%$ for various traffic densities. Various parameters showed in various scenarios are: number of selected vehicles, rates of timely informed neighboring nodes, traffic density and total number of informed vehicles.

Tables 1 and 2 show clearly that all nearby vehicles are informed before reaching the danger area. The number of engaged relays varies according to the speed and lifetime of the risk, which demonstrates the flexibility of EBP. These simulation results demonstrate the efficiency and the flexibility of the EBP, irrespective of the nature of the risk.

Figure 4 shows that "stop" message can work reliably as an acknowledgment of the next relay. This guarantees that a unique vehicle is selected to ensure rebroadcast of the warning message. It also avoids flooding of the network by warning messages and reduces the congestion phenomenon.

Under various network densities, EBP provides the best results thanks to sending of the "stop" message, which is a way to reduce the rate of alerts issued between the vehicles and, more specifically, to avoid the network overload.

Figure 5 and Figure 6 show that EBP offers better performance compared to both AG and Ocast protocols. Note that the Ocast protocol has been compared in [4], [5], [6], [18] with many existing protocols such as AG and was shown to have better performance.

In summary, using simulation experiments, we demonstrated that EBP can provide timely delivery of warning messages to all concerned vehicles, especially to those near the mobile risk. To improve its performance, EBP considers the lifetime of a message as well as mobility and direction of vehicles in the network. The main advantages of the proposed EBP protocol over the existing protocols are:

- reduction of congestion

- reduction of the number of transmitted messages in the network

- elimination of redundant warning messages
- identification and elimination of broken links by using Query/Response, which also preserves warning message from loss and fragmentation

- reduction of propagation time by sending only a single message for each broadcast cycle.

Therefore, this protocol can guarantee more effective security and safety information dissemination to all the neighbors in real time.

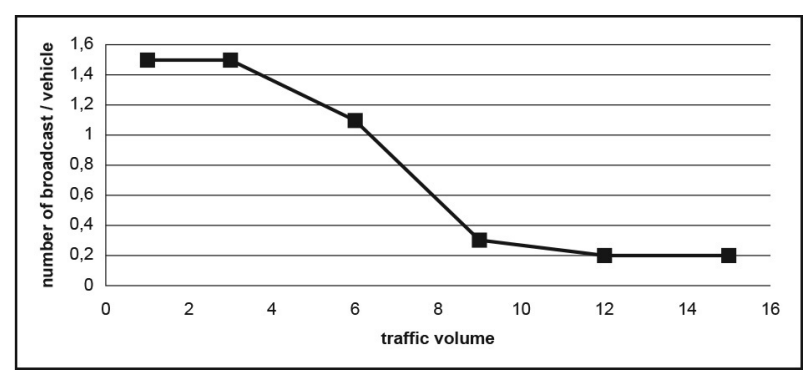

Figure 4. Number of broadcasts vs. traffic volume.

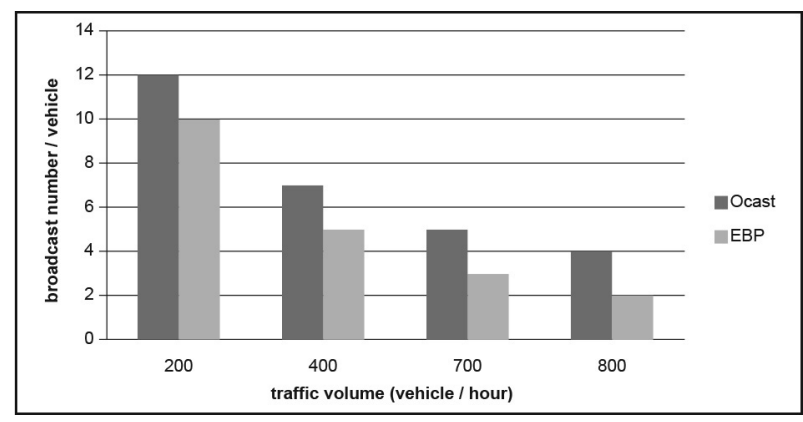

Figure 5. OCast vs EBP: variation of the broadcasts number in terms of traffic volume.

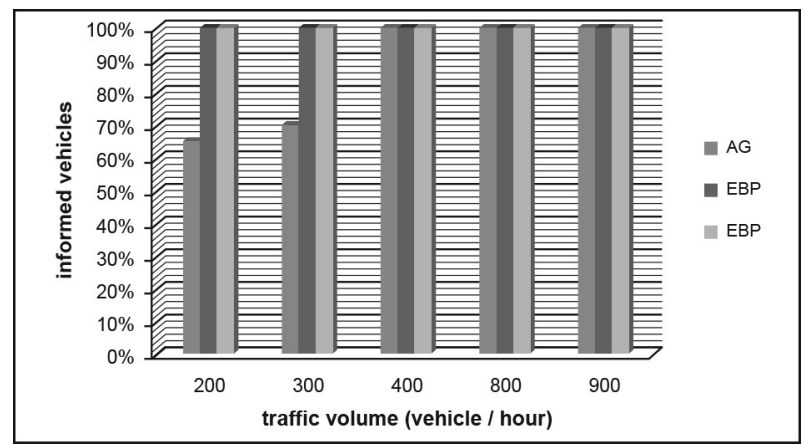

Figure 6. Informed vehicles vs. traffic volume for $\mathrm{AG}$, OCast and EBP. 


\section{Conclusion}

A new optimized protocol for warning messages dissemination, which is called Efficient Broadcast Protocol for Warning Messages in VANETs (EBP), is proposed in this paper. This protocol is especially suitable for VANETs in highway environments where the risk may have a mobile nature. It is developed based on in-depth analyses of the existing approaches and mechanisms used for data dissemination in VANETs. Efficiency of this protocol is validated using the simulation tool OMNET++ with plugins SUMO and Veins. Compared with other similar protocols, EBP offers the best results and performances in terms of warning messages delivery ratio. EBP can enhance road safety services in VANETs. EBP could be used in various risks situations, especially if the risk is mobile.

\section{References}

[1] S. H. Cha, "A Survey of Broadcast Protocols for Vehicular Ad-hoc Networks", SmartCR, vol. 4, no. 4, pp. 246-255, 2014.

https://doi.org/106029/smartcr.2014.04.001

[2] O. Mahma and A. Korichi, "Towards a New Approach of Data Dissemination in VANETS Networks", in Sixth Int. Conference on Computer Science, Engineering and Applications, Dubai, UAE, 2016, pp. 13-23.

https://doi.org/10.5121/csit.2016.60202

[3] S. Panichpapiboon and W. Pattara-atikom, "A Review of Information Dissemination Protocols for Vehicular Ad Hoc Networks", in IEEE Communications Surveys and Tutorials, 2011, vol. 14, no.3. pp. 784-798.

https://doi.org/10.1109/SURV.2011.070711.00131

[4] O. Mahma and A. Korichi, "Simulation \&VANET: Towards a New Reliable and Optimal Data Dissemination Model", IJANS, vol. 6, no. 2, 2016. https://doi.org/10.5121/ijans.2016.6201

[5] O. Mahma and A.Korichi, "Towards a Reliable Election Algorithm for VANETs Networks", IJCSIT, vol. 8, no 2, 2016.

https://doi.org/10.5121/ijcsit.2016.8209

[6] G. Korkmaz et al., "Urban Multi-hop Broadcast Protocol for Inter-vehicle Communication Systems", in ACM International Workshop on Vehicular Ad Hoc Networks, New York, NY, USA, 2004.

https://doi.org/10.1145/1023875.1023887
[7] G. Korkmaz et al., "An Efficient Fully Ad-Hoc Multi-Hop Broadcast Protocol for Inter-Vehicular Communication Systems", in IEEE Int'l Conf.on Communications, Istanbul, Turkey, 2006. https://doi.org/10.1109/ICC.2006.254764

[8] Q. Yang and L. Shen, "A Multi-hop Broadcast Scheme for Propagation of Emergency Messages in VANET", in Proc. of IEEE Int'l Conf. on Comm. Technology (ICCT), Nanjing, China, 2010, pp. 1072-1075.

https://doi.org/10.1109/ICCT.2010.5688509

[9] M. Zorzi and R. Rao, "Geographic Random Forwarding $(\mathrm{GeRaF})$ for Ad hoc and Sensor Networks: Energy and Latency Performance", in IEEE Trans. on Mobile Computing, vol. 2, no. 4, pp. 349-365, 2003.

https://doi.org/10.1109/TMC.2003.1255650

[10] Y. T. Yang and L. D. Chou, "Position-Based Adaptive Broadcast for Inter-vehicle Communications", in IEEE Int. Conf. on Communications Workshops, Beijing, China, 2008, pp. 410-414, 19-23.

https://doi.org/10.1109/ICCW.2008.83

[11] E. Fasolo et al., "Smart Broadcast Algorithm for Inter-vehicular Communications", in (WPMC), Padova, Italy, Tech. Rep, Dept. Inf, 2005.

[12] D. Li et al., “A Distance-based Directional Broadcast Protocol for Urban Vehicular Ad hoc Network", in Proc. of IEEE Int. Conf. on Wireless Communications, Networking and Mobile Computing (WiCom), Shanghai, China, 2007, pp. 1520-1523.

https://doi.org/10.1109/WICOM.2007.383

[13] S. Khakbaz and M. Fathy, "A Reliable Method for Disseminating Safety Information in Vehicular Ad hoc Networks Considering Fragmentation Problem", in Proc. of IEEE Int. Conf. on Wireless and Mobile Communications (ICWMC), Athens, Greece, 2008, pp. 25-30. https://doi.org/10.1109/ICWMC.2008.74

[14] M. Taha and Y. Hasan, "VANET-DSRC Protocol for Reliable Broadcasting of Life Safety Messages", in Proc. of IEEE Int. Symp. on Signal Processing and Information Technology, Giza, Egypt, 2007, pp. 104-109. https://doi.org/10.1109/ISSPIT.2007.4458046

[15] T. Osafune et al., "Multi-hop Vehicular Broadcast (MHVB)", in Proc. of IEEE 6th Int. Conf. on ITS Telecommunications (ITST), Chengdu, China, 2006, pp. 757-760.

https://doi.org/10.1109/ITST.2006.289011

[16] L. Ming et al., “Oppcast: Opportunistic Broadcast of Warning Messages in Vanets with Unreliable Links", in IEEE 6th Int'l Conf. on Mobile Adhoc and Sensor Systems (MASS'09), Macau, China, 2009, pp. 534-543.

https://doi.org/10.1109/MOBHOC.2009.5336959 
[17] Y. Qiangyuan and H. Geert, "Abiding Geocast for Warning Message Dissemination in Vehicular Ad Hoc Networks", in IEEE Int'l Conf. on Communications Workshops (ICC), Beijing, China, 2008, pp. 400-404. https://doi.org/10.1109/ICCW.2008.81

[18] A. Benaidja et al., "An Optimal Broadcast of Warning Messages in Vehicular Ad Hoc Networks", Int. J. of Computer and Information Technology, vol. 2, no. 5, pp. 986-992, 2013. https://doi.org/10.1.1.403.9237

[19] H. Alshaer and E. Horlait, "An Optimized Adaptive Broadcast Scheme for Inter-vehicle Communication", in IEEE 61st Conf. on Vehicular Technology (VTC 2005), Stockholm, Sweden, 2005, pp. $2840-2844$.

https://doi.org/10.1109/VETECS.2005.1543865

[20] H. Jiang et al., "Reliable and Efficient Alarm Message Routing in VANET", in Proc. of the 28th Int. Conf. on Distributed Computing Systems Workshops (ICDCSW'08), Beijing, China, 2008, pp. 186-191. https://doi.org/10.1109/ICDCS.Workshops.2008.34

[21] A. Shereen et al., "Survey on Broadcasting in VANETs", Research J. of Applied Sciences Engineering and Technology, vol. 07, no. 18, pp. 3733-3739, 2014. https://doi.org/10.19026/rjaset.7.728

[22] S. Panichpapiboon and G. Ferrari, "Irresponsible Forwarding", 8th Int. Conf. on ITS Telecommunications, Phuket, Thailand, 2008, pp. 311-316. https://doi.org/10.1109/ITST.2008.4740277
Received: December 2017 Revised: June 2018 Accepted: July 2018
Contact addresses:

Ouafa Mahma

Kasdi Merbah University

Ouargla, Algeria

e-mail: mahma.wafa@univ-ouargla.dz

Ahmed Korichi

Kasdi Merbah University

Ouargla, Algeria

e-mail: ahmed.korichi@univ-ouargla.dz

Abdelhabib Bourouis

ReLa(CS)2 Lab

University of Oum El Bouaghi

Algeria

e-mail: a.bourouis@univ-oeb.dz

Oufa Mahma is a PhD student in Computer Science at the Kasdi Merbah University, Ouargla (Algeria). She obtained both her Licence (BSc) and Master (MSc) degrees in Computer Science from the University of Ouargla in 2006 and 2009, respectively. She is a Graduate Teaching Assistant at the University of Ouargla since 2012 and head of IT administrative service at the Faculty of Natural Sciences at the University of Ouargla. Her doctoral research project focuses on efficient safety data dissemination in Vehicular Ad Hoc Networks.

Ahmed Korichi received his engineer degree in Computer Science from the University of Batna (Algeria) in 1987 and his Master and PhD degrees in Computer Science from the University of Batna, Algeria in 2001 and 2009, respectively. He obtained his "HDR" degree from the University of Biskra, Algeria in 2010. He joined the Department of Computer Science and Information Technology at the University of Ouargla, Algeria. Since 2001, Dr. Ahmed Korichi has been conducting research in the areas of network design and performance, modelling and simulation, QoS, wireless ad hoc networks, and vehicular networks. He has reviewed contributions in several scientific journals.

ABDElHabiB Bourouis received his "Ingénieur" degree in Computer Science from the University of Constantine (Algeria) in 1999, his "Magister" and "Doctorat en Sciences" degrees in Computer Science in 2003 and 2011, respectively, both from the University of Batna (Algeria). He obtained his "HDR" degree from the University of Biskra in 2015. He is "Maitre de Conférences A" at the University of Oum El Bouaghi (A1geria) since 2003. His research interests include artificial intelligence, model driven engineering, cryptology, performance evaluation, parallel and distributed simulation. 